\title{
17 Ziele: \\ SDG-Fortbildung für Lehrkräfte in Bremen
}

Die Agenda 2030 ist der internationale Aktionsplan für nachhaltige Entwicklung. In ihren 17 Zielen, den Sustainable Development Goals, finden sich die Vorgaben, um bis 2030 weltweit „die transformativen Schritte zu unternehmen, die dringend notwendig sind, um die Welt auf den Pfad der Nachhaltigkeit und der Widerstandsfähigkeit zu bringen“ (Agenda 2030).

Bremen bekennt sich zu diesen Zielen und arbeitet daran, die Menschen im Land über die globalen Herausforderungen zu informieren und zur Veränderung des eigenen Handelns zu motivieren. Die Diversität und Anzahl der Ziele mag auf den ersten Blick erschrecken und kann dazu führen sich auf einzelne zu fokussieren.

Diese Fortbildung, organisiert und durchgeführt vom Der Bevollmächtigte beim Bund und dem Landesinstitut für Schule Bremen, ist so gestaltet, dass die 17 Ziele erlebbar werden, Reflexion angeregt wird und Motivation zur Verhaltensänderung und zur Multiplikation des Erlebten und Erlernten entsteht. „17 Ziele - Eine Fortbildung“ betrachtet alle Ziele ganzheitlich und macht deutlich, dass alle Ziele in ihren $\mathrm{Zu}$ sammenhängen und auch Widersprüchen gleich wichtig sind.

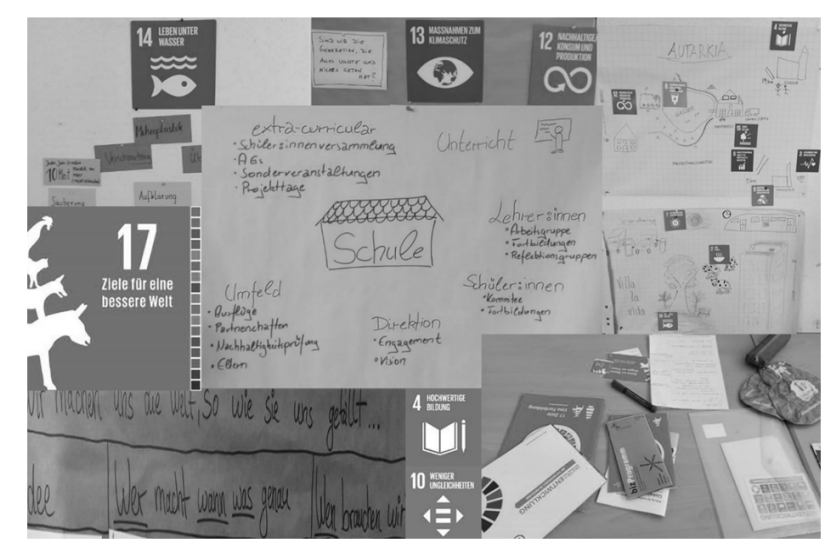

Abb. 1: Einblick in die Fortbildung, Quelle: Kroeger \& Froböse
Mit einem Fokus auf den Raum Schule als Bildungsort und Teil gesellschaftlichen Miteinanders erhalten die Teilnehmenden niedrigschwellige Methoden, um diese mit Schüler*innen und Kolleg*innen anzuwenden. Über das Herstellen eines direkten individuellen Bezugs anhand persönlicher Lebenswünsche und Vorstellungen werden die 17 Ziele greifbar und nachvollziehbar. Dadurch gelingt der Blick auf die Welt als Ganzes und motiviert, die Umsetzung der Agenda $2030 \mathrm{zu}$ unterstützen. „Es tut gut zu wissen, dass sich auch andere für die Verbesserung unseres Planeten einsetzen“ (Zitat Teilnehmende). Unterschiedliche Methoden, die mit verschiedenen Gruppen einfach anzuwenden sind, erleichtern den Zugang und die gemeinschaftliche Auseinandersetzung mit den Sustainable Development Goals und motivieren, Erlebtes und Gelerntes in die Schule zu bringen.

Die kritische Auseinandersetzung um die Bedeutung einzelner Ziele und ihrer Unterziele führt dazu, Ideen für nachhaltiges Handeln in der Schule und für den Alltag zu entwickeln und mitzunehmen. „Alles lässt sich für den Unterricht einsetzen und in Unterlagen nachschlagen - die genutzten Methoden haben den Inhalt verständlich transportiert" (Zitat Teilnehmender). Bereits in der Fortbildung werden gemeinsam Schritte geplant, um nachhaltige Entwicklung im Sinne der Agenda 2030 nicht nur im Unterricht, sondern auch in der Schule zu verankern.

Die Fortbildung endet nach eineinhalb Tagen, die gemeinsame Arbeit hat jedoch gerade erst begonnen. Neben Materialien und Methoden für den Unterricht wird den Kolleg*innen Beratung und Unterstützung angeboten.

Timm Kroeger, inhaltliche Konzeption und Durchführung, Mitarbeiter bei Der Bevollmächtigte beim Bund Bremen Claudia Froböse, Referentin für politische Bildung beim Landesinstitut für Schule Bremen doi.org/10.31244/zep.2019.04.07 